Trauma Berufskrankh 2006 · [Suppl 1]: S67-S73 DOI 10.1007/s10039-006-1112-4

Online publiziert: 15. März 2006

(c) Springer Medizin Verlag 2006

N. P. Südkamp ${ }^{1} \cdot$ J. Angenendt ${ }^{2} \cdot$ H. Hecht ${ }^{2} \cdot$ U. Nowotny-Behrens ${ }^{2} \cdot$ W. Köstler ${ }^{1}$. P. Niemeyer ${ }^{1} \cdot$ B. Stark ${ }^{3} \cdot$ M. Berger ${ }^{2}$

${ }^{1}$ Department für Orthopädie und Unfallchirurgie, Universitätsklinikum, Freiburg

${ }^{2}$ Abteilung für Psychiatrie und Psychotherapie, Universitätsklinikum, Freiburg

${ }^{3}$ Abteilung für Plastische Chirurgie und Handchirurgie, Universitätsklinikum, Freiburg

\title{
Freiburger Arbeitsunfallstudie (FAUST)
}

\section{Teil l: Epidemiologie und Verlauf psychischer Folgestörungen nach Arbeitsunfällen}

Freiburger Forschergruppe, die seit Mitte der 1990er Jahre zunächst bei Verkehrs- und später bei Arbeitsunfallopfern durchgeführt wurden. In Kooperation mit der unfallchirurgischen Abteilung des Universitätsklinikums Freiburg wurden 350 Verletzte wenige Tage nach ihrem Unfall mit Hilfe einer multimodalen diagnostischen Erhebung auf ihr peritraumatisches Erleben, ihre nachfolgenden psychischen Reaktionen und ggf. Störungen hin untersucht $[6,7]$. Ausgehend von diesen Ergebnissen wurden aus der Vielzahl der untersuchten prä-, peri- und posttraumatischen Variablen retrospektiv Risikomerkmale ermittelt, mit deren Hilfe das Auftreten einer posttraumatischen Belastungsstörung (PTBS) 6 Monate später vorhergesagt werden konnte. Es wurde ein 10 dichotome Items umfassender Screeningbogen konstruiert, der mit einer Sensitivität von $89 \%$ und einer Spezifität von $70 \%$ das spätere Auftreten einer PTBS vorhersagen konnte [11].

Die weite Verbreitung unfallbedingter psychischer Gesundheitsstörungen einerseits, ihre beträchtlichen Folgen sowie das Vorhandensein eines Instruments zur frühzeitigen Identifikation von Patienten mit erhöhtem Risiko für die spätere Entwicklung einer PTBS waren die zentralen Ausgangspunkte für die Konzeption der aktuellen Studie. Sie enthält einen epidemiologischen und einen Interventionsteil. Letzterer wird von Angenendt et al. in diesem Heft schwerpunktmäßig vorgestellt.
Ziel der vorliegenden Studie waren - die Identifikation prämorbider Einflussfaktoren (soziodemografische Variablen, frühere Unfälle, vorbestehende psychische Störungen), welche die Entwicklung psychischer Störungen nach Arbeitsunfällen begünstigen, sowie

- die Identifikation psychischer Belastungen, die neben den körperlichen Faktoren den Heilungsprozess mit beeinflussen.

Vollständige Auflistung der an der Studie beteiligten Institutionen und Personen: Forschergruppe des Universitätsklinikums Freiburg:

Abteilung für Psychiatrie und Psychotherapie (Prof. Dr. M. Berger): Mathias Berger,

Jörg Angenendt, Heide Hecht, Ute Nowotny-Behrens, Ullrich Frommberger Klinik für Traumatologie im Department Orthopädie und Traumatologie (Prof. Dr. N. Südkamp): Norbert Südkamp, Wolfgang Köstler,

Babette Röhrich, Philipp Niemeyer Abteilung für Plastische und Handchirurgie (Prof. Dr. B. Stark): Björn Stark, Horst Zajonc, Beate Munder

Unter Mitwirkung der Rekrutierungs- und Behandlungszentren:

St.-Josephs-Krankenhaus, Freiburg

(Prof. Dr. W. Schlickewei)

Klinikum Offenburg, Abteilung für Unfallchirurgie (Prof. Dr. J. Rudigier): Vanilla Nguyen, Brigitte Anders, Esther Dame

BG-Unfallklinik Tübingen (Prof. Dr. K. Weise): Andreas Badke, Marcel Pappon,

Alexander Breitung

BG-Unfallklinik Murnau (Prof. Dr. V. Bühren): Johanna Arneth-Graf, Karin Bauer, Astrid Bühren
Wichtige Vorarbeiten zu ihr bildeten die wissenschaftlichen Untersuchungen der 


\section{Arbeitsunfall und psychische Gesundheitsschäden}

Screening-Fragebogen zu Beschwerden nach einem Unfall

Name:

Alter:

Geschlecht:
Station:

heutiges Datum:

Unfalltag:
Sie haben vor wenigen Tagen einen Unfall erlitten. Viele Menschen haben nach einem solchen Ereignis nicht nur körperliche Beschwerden erlitten, sondern leiden auch seelisch unter den Folgen des Unfalls. Hier ist eine Liste von Beschwerden, die nach einem Unfall häufig beobachtet werden. Um Ihnen besser helfen zu können, kreuzen Sie bitte bei den nachfolgenden Fragen an, ob Sie seit dem Unfall unter den angegebenen Problemen gelitten haben. Wichtig ist, dass diese Beschwerden vor dem Unfall nicht vorhanden waren.

1. Hatten Sie während des Unfalls oder kurz danach starke Angst (oder evtl. sogar Todesangst? ja O nein $\mathrm{O}$

2. Fühlten Sie sich während des Unfalls oder kurz danach hilflos?

$$
\text { ja O nein } \mathrm{O}
$$

3. Fühlen Sie sich psychisch sehr belastet, wenn Sie etwas sehen oder hören, das Sie an den Unfall erinnert? ja O nein $\mathrm{O}$

4. Spüren Sie Wellen von starken Gefühlen in Zusammenhang mit dem Unfall (Aufgewühltsein oder Unwohlsein)?

$$
\text { ja } \mathrm{O} \text { nein } \mathrm{O}
$$

5. Fällt es Ihnen schwer einzuschlafen oder durchzuschlafen, weil Ihnen Bilder oder Gedanken über den Unfall in den Sinn kommen?

ja O nein $\mathrm{O}$

6. Haben Sie plötzlich auftretende Bilder vom Unfall in Ihrem Kopf?

$$
\text { ja } \mathrm{O} \text { nein } \mathrm{O}
$$

7. Haben Sie belastende Träume oder Alpträume vom Unfall? ja $\mathrm{O}$ nein $\mathrm{O}$

8. Vermeiden Sie über den Unfall nachzudenken?

$$
\text { ja O nein O }
$$

9. Haben Sie den Eindruck, dass Ihre Fähigkeit Gefühle zu erleben, eingeschränkt ist (sich abgestumpft fühlen)?

$$
\text { ja O nein } \mathrm{O}
$$

10. Befürchten Sie schlimme Folgen für Ihre Gesundheit (u.a. durch körperliche Behinderung)? ja $\mathrm{O}$ nein $\mathrm{O}$

Abb. $1 \Delta$ Screeningfragebogen zu Beschwerden nach einem Unfall, nach Stieglitz et al. [11]

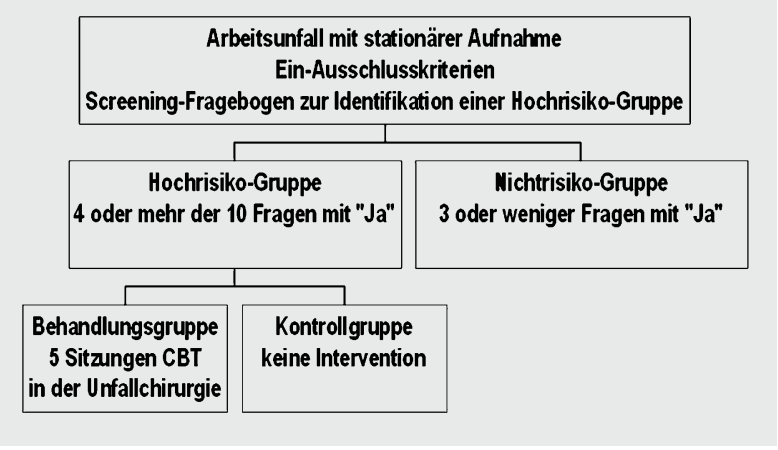

Abb. $2<$ Studiendesign: Einschluss sämtlicher Patienten in die Studie unabhängig vom Studienzweig innerhalb von 28 Tagen nach dem Unfallereignis; Verlaufsmessungen 4-malig in allen Gruppen bis zu 15 Monate nach dem Unfallereignis

Im Rahmen dieser Arbeit werden der kurz- und langfristige Verlauf psychischer Reaktionen und Störungen in der Nichtund der (unbehandelten) Hochrisikokontrollgruppe beschrieben.

\section{Studiendesign und Untersuchungsmethoden}

\section{Studienaufbau}

Die Patientenrekrutierung erfolgte ab März 2001 in den Abteilungen für Unfallchirurgie sowie Plastische- und Handchirurgie des UKL Freiburg, der Abtei- lung für Unfallchirurgie des St.-Josephs-Krankenhaus Freiburg, dem Klinikum Offenburg, den BG-Unfallkliniken in Murnau und Tübingen. In den beteiligten Zentren wurden konsekutiv alle Patienten der UV-Träger bis zum 30.09.2003, die stationär behandlungspflichtig waren, von Projektmitarbeitern über die Studie informiert und anhand des Screeningbogens ( Abb. 1) in Nicht- und Hochrisikogruppe eingeteilt. Gemäß dem Studiendesign (• Abb. 2) erfolgte innerhalb der Hochrisikogruppe eine Randomisierung in den Interventions- oder Kontrollarm. Zur Ergänzung der Gruppen wurden Patienten anderer Kostenträger (gesetzliche Krankenversicherung, private Unfall- oder Krankenversicherung) in die Studie aufgenommen, wenn das Unfallgeschehen dem eines Arbeits- oder Wegeunfalls vergleichbar war.

Folgende Kriterien mussten für einen Einschluss in die Studie gegeben sein:

- Abstand zum Unfallereignis weniger als 28 Tage

- Volljährigkeit des Patienten

- Ausreichende deutsche Sprachkenntnisse

- Vorliegendes informiertes schriftliches Einverständnis

Als Ausschlusskriterien wurden definiert:

- aktuelle intensivmedizinische Behandlung

- Schädel-Hirn-Trauma

- Querschnittverletzungen

- Vorliegen einer schizophrenen oder bipolaren Störung

- Vorliegen einer Alkoholabhängigkeit

- Notwendigkeit einer sofortigen psychiatrisch-psychotherapeutischen Behandlung

- Wohnort mit mehr als 50 km Entfernung zum Rekrutierungszentrum

\section{Gruppeneinteilung}

Nach Überprüfung der Ein- und Ausschlusskriterien und Vorgabe des Screeninginstruments erfolgte die Zuweisung zur Nicht- bzw. Hochrisikogruppe gemäß des in Abb. 2 dargestellten Screeningbogens. Patienten mit einem Wert $\geq 4$ Punkten wurden der Hochrisikogruppe (HR) zugeordnet; Patienten mit einem Punktwert $<4$ der Nichtrisikogruppe (NR). 
In der NR-Gruppe erfolgten lediglich 4-malige diagnostische Untersuchungen im Abstand bis maximal 15 Monate; die Patienten der HR-Gruppe wurden nach einem von einem Biomathematiker a priori erstellten Stratifizierungsplan randomisiert der Kontroll- (HR-K) oder der Interventionsgruppe (HR-VT) zugeteilt.

In der Kontrollgruppe wurde wie in der Nichtrisikogruppe verfahren (s. oben). Nur die Patienten der Therapiegruppe erhielten eine Frühintervention.

\section{Datenerhebung}

Alle Patienten wurden mit Hilfe von Unfallinterviews, die von der Arbeitsgruppe, basierend auf Hypothesen aus der Forschungsliteratur und eigenen Vorarbeiten, entwickelt worden waren, befragt. Der Interviewleitfaden enthält Fragen

- zum Unfallgeschehen,

- zum subjektiven Erleben des Unfalls und der Erstversorgung,

- zur Antizipation der Unfallfolgen und der psychosozialen Unterstützung (bei Erstbefragung).

Bei den Nachuntersuchungen bestand das Interview aus Fragen

- zum Verlauf des weiteren medizinischen, psychischen und psychosozialen Heilungsprozesses,

- zur beruflichen Rehabilitation,

- zur subjektiven Zukunftsperspektive usw.

Weiterer Bestandteil der Datenerhebung bei Einschluss in die Studie und den Katamneseuntersuchungen war das standardisierte diagnostische Interview (DIA-X) [13], mit welchem computergestützt psychische Störungen gemäß DSM-IV- bzw. ICD-10-Kriterien erfasst werden. Neben der PTBS werden auch andere psychische Störungen detektiert, die im Sinne häufiger komorbider Störungen nach Traumen von Bedeutung sind. Die Diagnosen können für die letzten 6 bzw. 12 Monate und für die Lebenszeit erhoben werden.

Zur Dokumentation der chirurgischen Diagnosen wurde auf die Krankenakten zurückgegriffen. Anhand der vorliegenden ICD-10-Diagnosen wurde durch einen Facharzt für Chirurgie ein international gebräuchliches Maß der Verletzungs-

Trauma Berufskrankh 2006 - 8 [Suppl 1]: S67-S72 DOI 10.1007/s10039-006-1112-4

(c) Springer Medizin Verlag 2006

N. P. Südkamp · J. Angenendt · H. Hecht · U. Nowotny-Behrens · W. Köstler · P. Niemeyer · B. Stark $\cdot$ M. Berger

Freiburger Arbeitsunfallstudie (FAUST). Teil I: Epidemiologie und Verlauf psychischer Folgestörungen nach Arbeitsunfällen

\section{Zusammenfassung}

Psychische Gesundheitsstörungen nach Arbeitsunfällen stellen ein unterschätztes Problem dar. Im Rahmen einer multizentrischen Studie wurden 408 Patienten, die nach Arbeitsunfällen stationär chirurgisch behandelt werden mussten, mittels Screeningbogen in eine Nichtrisiko- (NR) und eine Hochrisikogruppe (HR) eingeteilt und auf ihre nachfolgenden psychischen Reaktionen hin untersucht (4 Messzeitpunkte, 15 Monate Nachbeobachtung). Untersucht wurden Art, Schwere und Häufigkeit psychischer Störungen nach körperlichen Traumen in ihren spontanen Verläufen und ihren Auswirkungen auf die langfristige psychosoziale und berufliche Rehabilitation. Weibliches Geschlecht sowie eine positive Anamnese für mindestens eine psychiatrische Diagnose in der Vorgeschichte scheinen Risikofaktoren für die Zugehörigkeit zur HR-Gruppe zu sein. HR- und NR-Patienten unterschieden sich sowohl bei Studien-

\section{Freiburg Occupational Accidents Study (FAUST). Part I: Epidemiology and course of psychic disturbances consequent on occupational accidents}

\section{Abstract}

Both the frequency and the extent of mental health problems following accidents at the workplace are underdiagnosed. A prospective multi-centre study was conducted in 408 patients who needed to be admitted to hospital for surgical treatment after occupational accidents. These patients were allocated to a a no-risk (NR) and a high-risk (HR) group and examined on four occasions within 15 months for psychic sequelae of their injuries. Type, severity and duration of psychiatric disorder following trauma were investigated with respect to the spontaneous course and to their impact on the patients' longterm psychosocial and occupational rehabilitation. The data suggest that both female sex and at least one prior diagnosis of psychiatric illness are associated with $\mathrm{HR}$ status at study entry. Differences in many of the outcome variables were observed between the HR and NR groups shortly after trauma and beginn als auch im Verlauf in vielen Untersuchungsmerkmalen. Die Ergebnisse unterstreichen erneut die hohe Relevanz dieser Gesundheitsstörungen. Besonderer Handlungsbedarf ergibt sich eindeutig für die Gruppe der Hochrisikopatienten. In ihr zeigten sich über den kompletten Beobachtungszeitraum von 15 Monaten deutlich ungünstigere körperliche, psychische und soziale Heilungsverläufe. Auf diese spezielle, aber auch heterogene Untergruppe sollten sich die klinischen und wissenschaftlichen Bemühungen zur Entwicklung wirksamer Formen der psychologischen Akutbetreuung und Nachsorge konzentrieren.

\section{Schlüsselwörter}

Arbeitsunfall · Psychische Gesundheitsstörungen · Risikofaktoren · Heilungsverlauf · Psychologische Akutbetreuung persisted long after the event. The study results strongly re-emphasise the significance of such posttraumatic psychic reactions. It was obvious that action is needed especially for patients identified as belonging to the $\mathrm{HR}$ group, who were characterised by a less favourable course of recovery in physical, psychological and social terms throughout the 15-month follow-up period. Considerable scientific and clinical effort should be invested in developing effective psychological care for this distinct, albeit heterogeneous, subgroup of patients, to be applied both in the acute inpatient setting and during the subsequent outpatient phase.

\section{Keywords}

Occupational accident $\cdot$ Mental health problems · Risk factors · Course of recovery . Acute psychological care 


\section{Arbeitsunfall und psychische Gesundheitsschäden}

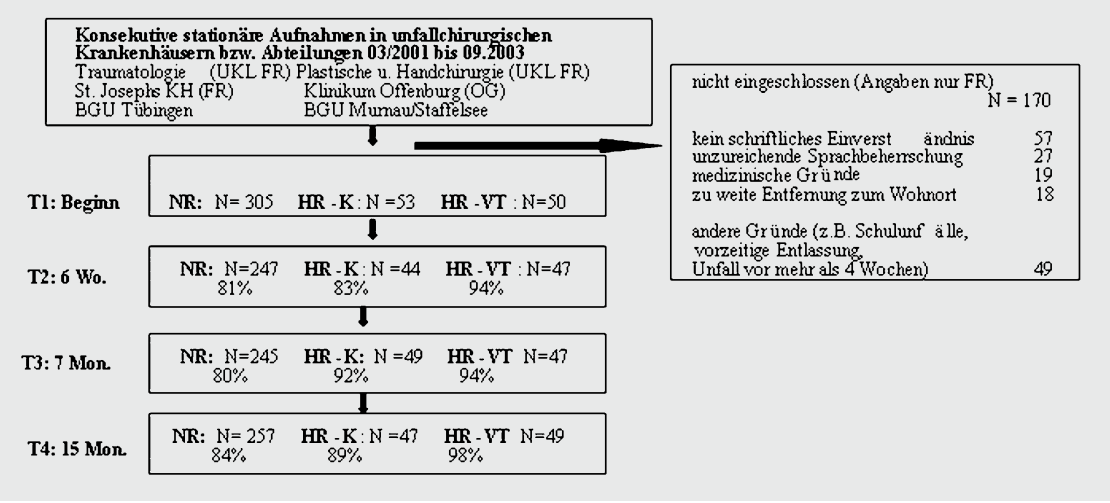

Abb. $3 \Delta$ Rekrutierung und Rücklauf im Rahmen der Nachuntersuchungen

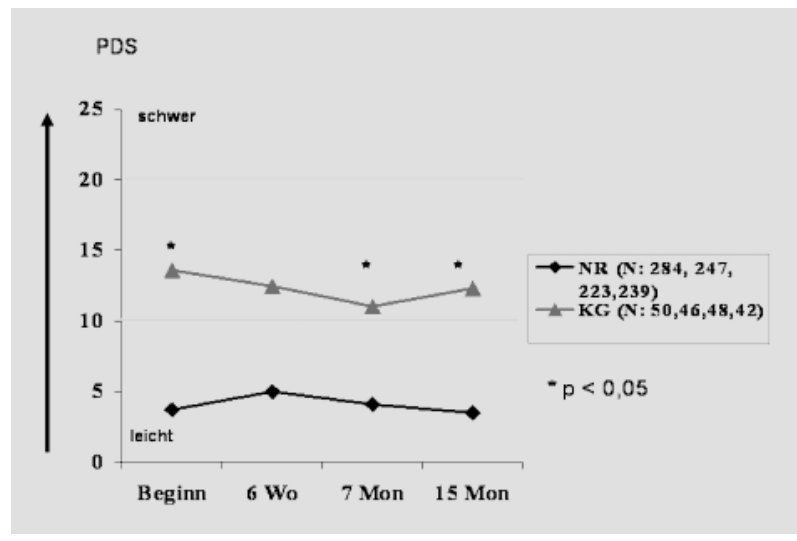

Abb. $4<$ PTBS-Symptomverlauf für unbehandelte Kontrollen (NR vs. HR-K)

schwere bestimmt (,injury severity scale“, ISS) $[2,3]$.

Die Verlaufsuntersuchung erfolgte zu 4 Messzeitpunkten bis zu 15 Monate nach dem Arbeitsunfall. Zur Datenerhebung wurden unterschiedliche Selbst- und Fremdbeurteilungsverfahren eingesetzt:

- Zur Erfassung der PTBS wurde die

PDS („posttraumatic diagnostic sca-

le") [4] verwendet. Der internatio-

nal eingesetzte Fragebogen enthält

17 Aussagen zur Häufigkeit der 3 zen-

tralen Symptomaspekte (intrusives

Wiedererleben, Übererregung und

Vermeidungsverhalten).

- Das BDI („Beck depressions-inventar") [8] erfasst die Intensität des depressiven Beschwerdesyndroms mit Hilfe von 21 Aussagen über typische Symptome depressiven Erlebens und Verhaltens.

- Zur Messung allgemeiner psychischer Beschwerden wurde die Symptomcheckliste (SCL-9o-R-Skala) [5] verwendet. In Form von 90 Aussagen werden 9 verschiedene psychische und psychovegetative Beschwerdebereiche (Ängstlichkeit, phobische
Angst, Unsicherheit, Somatisierung usw.) abgebildet.

Unabhängig von den Angaben der Patienten bei den Katamneseinterviews wurde für den Zeitpunkt der letzten Nachuntersuchung ein Bogen zur Erhebung objektiver Verlaufsdaten an die UV-Träger versandt. Dieser enthält 13 Fragen zur Dauer der stationären und ambulanten Heilbehandlung, zu den durchgeführten Maßnahmen der medizinischen und beruflichen Rehabilitation, zu Gutachten und sozialrechtlichen Aspekten (Minderung der Erwerbsfähigkeit: MdE), psychiatrisch-psychotherapeutischen Behandlungsmaßnahmen sowie eine Gesamteinschätzung des Heilungsverlaufs.

Die Fragen wurden für die unfallversicherten Patienten anhand der Verwaltungsakte von den Mitgliedsverwaltungen beantwortet. Für Patienten ohne UV-Status sind vergleichbare Daten der zuständigen Kostenträger (z. B. gesetzliche Krankenversicherung, private Krankenversicherung) nicht verfügbar.

\section{Datenauswertung und statistische Aufarbeitung}

Die Auswertung des Datenpools erfolgte entsprechend den jeweiligen Fragestellungen in Form deskriptiver und interferenzstatistischer Verfahren. Im Falle nominalskalierter Daten wurde über die 3 Vergleichsgruppen als Overall-Test ein $\chi^{2}$-Test gerechnet, im Fall intervallskalierter Daten eine ANOVA. Bei signifikantem Overall-Test wurden Unterschiede zwischen jeweils 2 Vergleichsgruppen mit dem $\chi^{2}$-Test bzw. mit dem T-Test gegen den Zufall abgesichert. Effektstärken, die sich auf die Gruppenzugehörigkeit und eine intervallskalierte Variable beziehen, wurden als Korrelationskoeffizient dargestellt. $\alpha<0,05$ wurde als Signifikanzgrenze festgelegt, $\alpha<0,10$ als Tendenz gewertet.

\section{Ergebnisse}

In der vorliegenden Arbeit werden die Resultate dargestellt, die sich auf die gesamte Untersuchungsstichprobe und deren Hauptmerkmale bei Studieneinschluss beziehen. Außerdem werden die Verlaufsergebnisse der Nicht- und Hochrisikogruppe ohne Frühintervention (HR-Kontrolle) einander gegenübergestellt.

In die Studie konnten 408 Patienten eingeschlossen werden. Gemäß dem Screeningfragebogen wurden 103 Patienten der Hoch- (HR), 305 Patienten der Nichtrisikogruppe (NR) zugeordnet. Die Rücklaufquote (gemessen am Vorhandensein von Interview und/oder auswertbarem PDS-Wert) in allen Untergruppen lag zu allen Messzeitenpunkten bei mindestens $80 \%$ (• Abb. 3).

Die untersuchte Gesamtstichprobe zeichnete sich (- Tab. 1) durch die folgenden Merkmale aus: Mit 83\% der Betroffenen überwog das männliche $\mathrm{Ge}$ schlecht, die Patienten waren im Durchschnitt 41 Jahre alt. Die Teilnehmer verfügten mehrheitlich über einen einfachen bis mittleren Bildungsabschluss, waren zum Zeitpunkt des Unfalls überwiegend als gelernte Arbeiter oder einfache bzw. mittlere Angestellte in Vollzeit beschäftigt. Mit Ausnahme des Geschlechts und der Lebensform waren in den 3 Untergruppen keine Unterschiede bezüglich soziodemografischer Merkmale festzustellen. In der 
Gruppe der HR-Patienten (Gesamtgruppe) war der Anteil von Frauen mit 26\% signifikant höher als in der NR-Gruppe (13\%). Ansonsten waren die soziodemografischen Merkmale für den weiteren Verlauf psychischer Gesundheitsstörungen nach Arbeitsunfällen ohne prognostische Bedeutung.

Da die Schwere der unfallbedingten Verletzungen als Einflussfaktor für psychische Folgebeschwerden bekannt ist, wurde diese differenziert erfasst, um sie ggf. bei weiteren Auswertungsschritten statistisch kontrollieren zu können. Die mittlere Verletzungsschwere ist mit einem ISS-Wert von 7,6 $(S D=6)$ als im Durchschnitt mittelschwer zu bewerten: Die Patienten der HR-Gruppe wiesen dabei signifikant schwerere Verletzungen auf als die der NR-Gruppe [Mittelwert $=9,9$ $(\mathrm{SD}=7,4)$ vs. Mittelwert=6,9 $(\mathrm{SD}=5,2)$; $p<0,05] .15$ Patienten mit ISS-Werten $>20$ wurden in die Studie eingeschlossen. Dieser geringe Anteil von Schwerstverletzten am Gesamtkollektiv ist auf den Ausschluss von intensivstationspflichtigen $\mathrm{Pa}$ tienten sowie von Patienten mit SchädelHirn-Trauma zurückzuführen.

\section{Vorbestehende psychische Störungen}

Bei der Erstbefragung wurde erhoben, wie häufig bei den Unfallopfern unter Berücksichtigung der gesamten Lebensspanne bereits vor dem Unfall psychische Störungen mit Krankheitswert aufgetreten waren. Dabei ergaben sich signifikante Unterschiede für die Häufigkeit ermittelter Lebenszeitdiagnosen zwischen den Gruppen (• Tab. 2). In der NR-Gruppe war zu irgendeinem Zeitpunkt vor dem Unfall bei 79 Unfallopfern (26\%) bereits mindestens eine psychische Störung vorhanden gewesen. In der Gruppe der HR (gesamt) hatten 45 Patienten (44\%) im bisherigen Verlauf die Diagnosekriterien für mindestens eine psychische Störung erfüllt. Als die häufigsten in der Längsschnittbetrachtung vorbestehenden $\mathrm{Di}$ agnosen wurden in beiden Gruppen ohne relevante Abweichungen Angststörungen (v. a. phobische Ängste), affektive Störungen (depressive Episode oder Dysthymie) und schädlicher Substanzgebrauch (v. a. Alkohol) festgestellt. Bei $4 \%$ (NR)

Tab. 1 Patienten- und Unfallcharakteristika nach Gruppenzuordnung

\begin{tabular}{|c|c|c|c|c|c|}
\hline Merkmal & NR & HR-K & HR-VT & $\begin{array}{l}\text { Statistische } \\
\text { Parameter }\end{array}$ & $\begin{array}{l}\text { NR vs. HR } \\
\text { (gesamt) }\end{array}$ \\
\hline $\begin{array}{l}\text { Soziodemografische Daten } \\
\text { (n) }\end{array}$ & 305 & 50 & 53 & & \\
\hline Männlich [\%] & 86,9 & 75,5 & 72,0 & $x^{2}$ & $\begin{array}{l}\text { Statistisch } \\
\text { signifikant }\end{array}$ \\
\hline $\begin{array}{l}\text { Alter } \\
\text { (Mittelwert } \pm \text { Standard- } \\
\text { abweichung) }\end{array}$ & $41,4 \pm 12,3$ & $41,2 \pm 11,5$ & $39,7 \pm 11,2$ & T-Test & n.s. \\
\hline $\begin{array}{l}\text { Mit Anderen zusammenle- } \\
\text { bend [\%] }\end{array}$ & 66,1 & 62,3 & 46,9 & $x^{2}$ & $\begin{array}{l}\text { Statistisch } \\
\text { signifikant }\end{array}$ \\
\hline (Fach)-Arbeiter [\%] & 53,5 & 51,9 & 50,0 & $x^{2}$ & n.s. \\
\hline Angestellter [\%] & 18,7 & 26,9 & 30,0 & & \\
\hline Sonstige [\%] & 27,8 & 21,2 & 20,0 & & \\
\hline Volltagsbeschäftigt (\%) & 83,2 & 78,0 & 79,2 & $x^{2}$ & n.s. \\
\hline \multicolumn{6}{|l|}{ Unfallbezogene Daten } \\
\hline $\begin{array}{l}\text { Verletzungsschwere (ISS) } \\
\text { Mittelwert } \pm \text { Standard- } \\
\text { abweichung }\end{array}$ & $6,87 \pm 5,2$ & $9,47 \pm 7,2$ & $10,4 \pm 7,6$ & $\begin{array}{l}\text { T-Test } \\
r_{\text {bis }}=0,22\end{array}$ & $\begin{array}{l}\text { Statistisch } \\
\text { signifikant }\end{array}$ \\
\hline
\end{tabular}

Tab. 2 Lebenszeitprävalenz psychischer Störungen vor dem Unfall

\begin{tabular}{|llllll}
\hline Merkmal & NR & HR-K & HR-VT & $\begin{array}{l}\text { Statistische } \\
\text { Parameter }\end{array}$ & $\begin{array}{l}\text { NR vs. HR } \\
\text { (gesamt) }\end{array}$ \\
Anzahl $(n)$ & 301 & 53 & 49 & & \\
\hline Mindestens 1 Lebenszeitdiagnose [\%] & 26,2 & 39,6 & 49,0 & $X^{2}$ & $\begin{array}{l}\text { Statistisch } \\
\text { signifikant }\end{array}$ \\
\hline $\begin{array}{l}\text { Diagnose bis 4 letzte Wochen vor Un- } \\
\text { fall anhaltend [\%] }\end{array}$ & 4,0 & 10,0 & 19,0 & $X^{2}$ & $\begin{array}{l}\text { Statistisch } \\
\text { signifikant }\end{array}$ \\
\hline
\end{tabular}

Tab. 3 Daten der UV-Träger

\begin{tabular}{|c|c|c|c|c|}
\hline Merkmal & NR & HR (gesamt) & $\begin{array}{l}\text { Statistische } \\
\text { Parameter }\end{array}$ & $\begin{array}{l}\text { Signifi- } \\
\text { kanz }\end{array}$ \\
\hline \multicolumn{5}{|l|}{ Verletzungsschwere ISS } \\
\hline Mittelwert \pm Standardabweichung & $6,87^{*} \pm 5,2$ & $9,92 \pm 7,4$ & T-Test & * \\
\hline \multicolumn{5}{|l|}{ Tage stationäre Erstbehandlung } \\
\hline Mittelwert \pm Standardabweichung & $15,5^{*} \pm 14,7$ & $26,3 \pm 34,3$ & T-Test & * \\
\hline Arbeitsunfähigkeit in Tagen & $139,2^{*} \pm 119,5$ & $193,6 \pm 149,0$ & T-Test & * \\
\hline Rückkehr in Unfallbetrieb [\%] & 85,1 & 72,9 & $x^{2}$ & * \\
\hline $\begin{array}{l}\text { Wiederaufnahme der alten Tätigkeit } \\
\text { [\%] }\end{array}$ & 82,3 & 64,3 & $x^{2}$ & * \\
\hline
\end{tabular}

Nur für Patienten mit geklärter UV-Zuständigkeit; $\mathrm{n}_{\max }=334$

*Statistisch signifikant

bzw. 15\% (HR) hatten diese Störungen bis innerhalb der letzten 4 Wochen vor dem Unfall angehalten (• Tab. 2).

\section{Verläufe der unbehandelten Kontrollgruppen (NR und HR-K)}

Die Bildung von Untergruppen auf der Basis des individuellen Screeningwerts dient der Identifizierung von Hochrisikopatienten und ist von hoher klinischer Relevanz.
HR-K- und NR-Patienten unterschieden sich bei Studienbeginn nicht nur bezüglich ihrer Screeningwerte kurz nach dem Unfall. Die Unterschiede bildeten sich auch in der Schwere des anfänglichen posttraumatischen Belastungssyndroms, des depressiven Syndroms und der allgemeinen psychischen Beschwerden ab. In der HR-K-Gruppe waren alle psychischen Symptomvariablen signifikant stärker ausgeprägt. Die durchschnittlichen Messwerte der NR blieben im Re- 
ferenzbereich „normaler Vergleichspersonen ohne psychische Störungen“" (BDI, SCL-90-R) bzw. im Bereich „sehr leichter“ Beschwerden (PDS).

In der Folgezeit blieben diese anfänglichen Unterschiede im Verlauf über die 3 weiteren Messzeitpunkte bis zu 15 Monate nach dem Unfall hinaus erhalten oder akzentuieren sich noch stärker. Bei der HRK-Gruppe war der spontane Beschwerdeverlauf der PTBS, der depressiven Symptomatik und der allgemeinen psychischen Belastung im Wesentlichen als Stagnation auf einem deutlich erhöhten Niveau zu beschreiben. Bei der letzten Nachuntersuchung waren die Ausprägungsgrade in allen untersuchten Symptombereichen im Durchschnitt vergleichbar mit den Ausgangswerten bei Studienbeginn. Im direkten Vergleich beider unbehandelter Gruppen ergeben sich somit signifikante Unterschiede zwischen den Gruppen für alle 3 untersuchten Symptomvariablen (exemplarisch für das posttraumatische $\mathrm{Be}$ lastungssyndrom in $\mathbf{A b b .} 4$ dargestellt).

Die beschriebenen Verlaufsunterschiede fanden sich in signifikant unterschiedlichen Anteilen von Patienten mit späteren psychischen Diagnosen wieder. 90\% der NR-Gruppe blieben im gesamten Nachuntersuchungszeitraum ohne diagnostizierte psychische Störung. Bei den unbehandelten HR-K-Patienten waren es im Vergleich nur 72\%. Mit zunehmender Dauer des Nachuntersuchungszeitraums war eine tendenzielle Abnahme manifester Störungen in beiden Gruppen zu beobachten. Langfristig ließen sich in der NR-Gruppe bei nur 12 (5\%) Patienten anhaltende psychische Störungen feststellen. Bei nur 3 Personen (1\%) der NRGruppe wurde zur letzten Nachuntersuchung die Diagnose einer PTBS gestellt.

In der HR-K-Gruppe wurden bei 9 Personen (20\%) anhaltende psychische Störungen festgestellt. Die Quote der unbehandelten HR-K-Patienten mit einer PTBS-Diagnose machte hier mit $17 \%$ (8 Patienten) den größten Anteil aus. Bei chronischem Verlauf der PTBS waren bei 2/3 dieser Fälle komplexe psychische Störungen mit mehr als einer Diagnose (Komorbidität) zu verzeichnen.

\section{Diskussion}

Die Ergebnisse der vorliegenden Studie demonstrieren, dass bei insgesamt etwa $17 \%$ der Gesamtstichprobe unfallchirurgischer Patienten innerhalb der ersten 15 Monate nach dem Arbeitsunfall psychische Störungen aufgetreten sind. Am häufigsten wurden Diagnosen der PTBS, von Angstund depressiven Störungen und Schmerzstörungen festgestellt. Das gleichzeitige Auftreten mehrerer Störungen war häufig und verweist auf die hohe Komplexität dieser psychischen Gesundheitsstörungen bei chronischem Verlauf.

Zwischen der Nicht-und der Hochrisikogruppe fanden sich bedeutende Unterschiede. Die identifizierten Risikopatienten (HR-VT und HR-K) entwickeln 3,5-mal häufiger psychische Störungen als Patienten der Nichtrisikogruppe. Die Quote bis zur letzten Katamnese anhaltender psychischer Störungen war sogar um den Faktor 4,7 erhöht. Bei einer Längsschnittbetrachtung, die bereits vor dem Unfall aufgetretene psychische Störungen in der Lebenszeitperspektive berücksichtigte, hatten die Risikopatienten auch zuvor schon deutlich häufiger psychische Störungen aufgewiesen. Vorbestehende - wenn auch wieder remittierte - psychische Störungen erhöhen demzufolge die Vulnerabilität für Belastungsstörungen nach einem Arbeitsunfall.

HR- und NR-Patienten unterschieden sich über den gesamten Studienzeitraum hinsichtlich einer Vielzahl von Merkmalen. Sie zeigten konsistente Ausgangsunterschiede bezüglich der anfänglichen Verletzungsschwere und der Dauer der chirurgischen Akutbehandlung sowie der Schwere ihrer psychischen Beschwerden bei der Ersterhebung. Beide Gruppen blieben im gesamten weiteren Heilungsverlauf klar unterscheidbar. Auch die spätere berufliche Wiedereingliederung, die langfristige psychische Symptombelastung, die Häufigkeit psychischer Störungen sowie die langfristige Alltagsbewältigung zeigten in der HR-Gesamtgruppe signifikant schlechtere Ergebnisse als in der NR-Gruppe (• Tab. 3). Die Studienresultate bestätigten somit die enorme klinische Relevanz einer frühzeitigen Identifizierung von Risikopatienten. Das verwendete kurze PTBS-Screening-Instru- ment ist dabei für eine handhabbare weil rasche und okönomische - Identifizierung von Risikopatienten zwar ein geeignetes Hilfsmittel, aber mit dem bisherigen Cut-off-Wert von 4 als alleiniges oder auch zentrales Kriterium einer Indikationsstellung zur Durchführung von psychologischen Frühinterventionsmaßnahmen nicht geeignet.

\section{Fazit für die Praxis}

Die Untersuchungsergebnisse zu den psychischen Folgen schwerer Arbeitsunfälle unterstreichen erneut die hohe Relevanz dieser Gesundheitsstörungen. Über das normale Ausmaß hinausgehende und zeitlich überdauernde psychische Beschwerden spielen vom Umfang und ihren langfristigen Folgen her eine nicht zu vernachlässigende Rolle. Selbst in dem Spektrum „mittelschwerer" unfallchirurgischer Verletzungen stellt sich die dringende Frage einer angemessenen Berücksichtigung der psychischen Unfallfolgen. Besonderer Handlungsund auch Behandlungsbedarf ergibt sich eindeutig für die Gruppe der Hochrisikopatienten. In ihr zeigten sich über den kompletten Beobachtungszeitraum von 15 Monaten hinweg deutlich ungünstigere körperliche, psychische und soziale Heilungsverläufe. Auf diese spezielle, aber auch heterogene Untergruppe sollten sich künftig die klinischen und wissenschaftlichen Bemühungen zur Entwicklung wirksamer Formen der psychologischen Akutbetreuung und Nachsorge konzentrieren.

\section{Korrespondierender Autor}

Prof. Dr. N. P. Südkamp

Department für Orthopädie und Unfallchirurgie, Universitätsklinikum

Hugstetter Straße 55, 79095 Freiburg

suedkamp@ch11.ukl.uni-freiburg.de

Interessenkonflikt. Es besteht kein Interessenkonflikt. Der korrespondierende Autor versichert, dass keine Verbindungen mit einer Firma, deren Produkt in dem Artikel genannt ist, oder einer Firma, die ein Konkurrenzprodukt vertreibt, bestehen. Die Präsentation des Themas ist unabhängig und die Darstellung der Inhalte produktneutral. 


\section{Literatur}

1. Arbeitskreis „Heilverfahren bei speziellen Verletzungsfolgen" (1999) Arbeitsunfall und psychische Gesundheitsschäden. Landesverband Südwestdeutschland der gewerblichen Berufsgenossenschaften, Heidelberg

2. Baker SP, O'Neill B (1976) The injury severity score: an update. J Trauma 16: 882-885

3. Baker SP, O'Neill B, Haddon W et al. (1974) The injury severity score: a method for describing patients with multiple injuries and evaluating emergency care. J Trauma 14: 187-196

4. Foa EB, Riggs DS, Dancu CV et al. (1993) Reliability and validity of a brief instrument for assessing post-traumatic stress disorder. J Traumatic Stress 6: 459-473

5. Franke GH (1995) Die Symptom-Checkliste von Derogatis -Deutsche Version. Beltz Test Gesellschaft, Göttingen

6. Frommberger UH, Stieglitz RD, Nyberg E et al. (1998) Prediction of posttraumatic stress disorder by immediate reactions to trauma: a prospective study in road traffic accident victims. Eur Arch Psychiatr Clin Neurosci 248: 316-321

7. Frommberger U, Stieglitz RD, Straub S et al. (1999) The concept of "sense of coherence" and the development of posttraumatic stress disorder in traffic accident victims. J Psychosom Res 46: 343-348

8. Hautzinger M, Bailer M, Worall H et al. (1992) Das Beck Depressionsinventar - BDI. Huber, Bern Stuttgart Toronto

9. Malt UF (1992) Coping with accidental injury. Psychiatr Med 10: 135-147

10. Schottenfeld RS, Cullen MR (1986) Recognition of occupation-induced posttraumatic stress disorders. J Occup Med 28: 365-369

11. Stieglitz RD, Nyberg E, Albert M et al. (2002) Entwicklung eines Screening-Instrumentes zur Identifizierung von Risikopatienten für die Entwicklung einer Posttraumatischen Belastungsstörung (PTB) nach einem Verkehrsunfall. Z Klin Psychol Psychother 31: 22-30

12. Weisaeth $L$ (1989) The stressors and the post-traumatic stress syndrome after an industrial disaster. Acta Psychiatr Scand [Suppl] 355: 25-37

13. Wittchen HU, Pfister H (1997) Diagnostisches Expertensystem (DIA-X) zur Diagnostik psychischer Störungen. Swets Test Services, Frankfurt 